\title{
Zürcher Gesundheitsgesetz: NEIN bleibt NEIN!
}

Mit 59 zu 41\% hat das Zürcher Stimmvolk die gesetzliche Änderung der Medikamentenabgabe verworfen. Vorgesehen war, diese nur noch jenen Ärzten zuzugestehen, die am allgemeinen Notfalldienst teilnehmen und in deren Gemeinde noch keine 24-Stunden-Apotheke tätig ist. Diese Regelung hätte zur Folge gehabt, dass allen Ärzten der Städte Zürich und Winterthur die Medikamentenabgabe per sofort verboten worden wäre. In jenen Gemeinden, in denen neue 24-Stunden-Apotheken eröffneten, spätestens nach Ablauf der Übergangsfrist.

Gegen dieses nicht sehr patientenfreundliche Gesetz haben die Zürcher Ärztinnen und Ärzte vor einem Jahr erfolgreich das Referendum ergriffen. Innert kürzester Zeit kamen damals 20000 Unterschriften zusammen. Trotz dieser breiten Unterstützung während der Phase des Referendums war die jetzige Ausgangslage keinesfalls rosig. Im Gegenteil, die Ärzteschaft hatte nicht nur gegen den Regierungsrat, die Mehrheit des Kantonsrates und die Apotheker zu kämpfen, sondern auch gegen die gesamte Zürcher Medienlandschaft. Eine politische Ausgangslage, die in ähnlicher Form bereits vor der Abstimmung im Jahre 2001 bestand.

Um so bedeutender ist der neuerliche Abstimmungserfolg zu werten. Das Volk schenkte den Argumenten der Ärzte Vertrauen und stimmte erneut gegen unnötige Einschränkungen der ärztlichen Medikamentenabgabe sowie gegen unbegründete wirtschaftliche Schutzmassnahmen zugunsten der Apotheken. Das Volk will einfach selbst entscheiden, wo es die Medikamente beziehen möchte. Ein Ergebnis, das ein Meinungsforschungsinstitut übrigens bereits nach der ersten Abstimmung analysiert hatte.

Dass dieser Abstimmungserfolg möglich wurde, verdanken wir vor allem dem beherzten Einsatz zahlreicher Ärztinnen und Ärzte. Sie haben sich eingebracht und überall im Kanton mit grossem Engagement zugunsten der Sache gekämpft. Nur so kann ein politischer Erfolg erzielt werden. Oder anders gesagt, politische Arbeit lässt sich nicht vollends delegieren. Sie ist eine permanente Aufgabe für alle am politischen Prozess beteiligten Akteure. Die Zürcher Ärztinnen und Ärzte haben gezeigt, dass sie diese Botschaft verstanden und verinnerlicht haben. Ihnen verdanken wir den Erfolg!

Das Referendum gegen die Revision des Krankenversicherungsgesetzes (KVG) sowie die anschliessende Volksabstimmung werden nun nicht nur die Zürcher, sondern alle Schweizer Ärztinnen und Ärzte beschäftigten. Sie können nun zeigen, dass sie ein gemeinsames politisches Ziel geschlossen verfolgen wollen. Die Zürcher Kolleginnen und Kollegen haben ihnen vorgemacht, wie dies geht. Unabdingbare Voraussetzung ist, dass alle am gleichen Strick ziehen und sich aktiv ins politische Geschehen einbringen.

Dr. rer. publ. HSG Sven Bradke Geschäftsführer der Ärzte mit Patientenapotheke (APA) 